\title{
Antitumor Activity of Eribulin After Fulvestrant Plus CDK4/6 Inhibitor in Breast Cancer Patient-derived Xenograft Models
}

\author{
YUKI NIWA $^{1}$, MAKOTO ASANO ${ }^{1}$, TAKAYUKI NAKAGAWA ${ }^{1}$, \\ DAMIEN FRANCE ${ }^{2}$, TARO SEMBA ${ }^{1}$ and YASUHIRO FUNAHASHI ${ }^{1}$ \\ ${ }^{1}$ Tsukuba Research Laboratories, Eisai Co., Ltd., Ibaraki, Japan; \\ ${ }^{2}$ Oncodesign, Dijon, France
}

\begin{abstract}
Background/Aim: There is no established standard chemotherapy after administration of the combination endocrine plus CDK4/6 inhibitor therapy for luminal-type breast cancer. We used patient-derived xenograft (PDX) models to determine the antitumor activity of eribulin and capecitabine after endocrine therapy plus CDK4/6 inhibitor. Materials and Methods: We examined the antitumor activity of fulvestrant, palbociclib, eribulin, and capecitabine in 4 luminal-type breast cancer PDX models (OD-BRE-0188, -0438, -0450, -0745). In $O D$-BRE-0438, we determined the antitumor activity of chemotherapy after fulvestrant-palbociclib treatment. We also performed immunohistochemical analysis to explore the effects of treatment on E-cadherin in tumor tissues. Results: Fulvestrant, fulvestrant-palbociclib and chemotherapy had antitumor activity in the 4 PDX models. In OD-BRE-0438 (the most resistant to fulvestrant-palbociclib), eribulin had superior antitumor activity to capecitabine after fulvestrant plus palbociclib. Only eribulin tended to increase E-cadherin expression. Conclusion: Eribulin had superior antitumor activity to capecitabine after fulvestrant-palbociclib in the $O D$ BRE-0438 model.
\end{abstract}

Breast cancer (BC) is one of the most common cancers and is a major cause of death in women. About $70 \%$ of all BCs are estrogen-receptor (ER)-positive, and these patients are treated with endocrine therapies such as selective ER modulators (e.g. tamoxifen), selective ER degraders (e.g.

This article is freely accessible online.

Correspondence to: Yasuhiro Funahashi, Tsukuba Research Laboratories, Eisai Co., Ltd., 5-1-3 Tokodai, Tsukuba, Ibaraki, 3002635, Japan. Tel: +81 298475615, Fax: +81 298477614, e-mail: y-funahashi@hhc.eisai.co.jp

Key Words: CDK4/6 inhibitor, eribulin mesylate, luminal-type breast cancer, patient-derived xenograft model, reversal of epithelial-mesenchymal transition, preclinical trial. fulvestrant) and aromatase inhibitors. Recently, several clinical trials have demonstrated that adding selective cyclindependent kinase (CDK) 4/6 inhibitors, such as palbociclib, ribociclib and abemaciclib, to endocrine therapy prolongs progression-free survival in patients with ER-positive BC (15). These CDK4/6 inhibitors have been approved and are widely used worldwide. Although their approval has provided improved clinical benefits to $\mathrm{BC}$ patients, almost all $\mathrm{BC}$ patients treated with endocrine therapy plus CDK4/6 inhibitors acquire resistance and need to be treated with anticancer chemotherapy.

Eribulin mesylate (eribulin) is an inhibitor of microtubule dynamics. It is a fully synthesized analogue of the natural product halichondrin $\mathrm{B}$, which derives from the marine sponge Halichondria okadai $(6,7)$. Eribulin has characteristic tubulin-binding activity; it binds to microtubule ends and inhibits microtubule polymerization, thus causing cell-cycle arrest and inducing apoptosis (8). A pivotal phase III study (EMBRACE) has revealed that eribulin significantly improves overall survival (9), and it is currently used in many countries for late-stage, locally advanced or metastatic BC. Eribulin has not only mitotic activity but also non-mitotic activities such as vascular remodeling activity and reversal of epithelial-to-mesenchymal transition (EMT) $(10,11)$; these unique activities may contribute to its clinical benefits.

Since the approval of CDK4/6 inhibitors, their combination with endocrine therapies has been chosen to treat ER-positive BC patients in first line and subsequent therapies. However, there is no clinical data suggesting a standard treatment regimen against $\mathrm{BC}$ in patients who are resistant to these combined therapies. Here, following fulvestrant plus palbociclib combination treatment, we determined the antitumor activity of eribulin and capecitabine, which have been used in patients with ERpositive $\mathrm{BC}$ in the anticancer chemotherapy stages, by using luminal-type-BC patient-derived xenograft (PDX) models faithfully recapitulating human tumor biology (12). After treatment with the combination of fulvestrant plus a CDK4/6 inhibitor followed by eribulin or capecitabine, E-cadherin 
expression on the cell membrane was determined by immunohistochemical analysis, and the differences in cellmembrane E-cadherin expression in eribulin- and capecitabine-treated tumors were analyzed. This preclinical study adds to the preclinical evidence supporting treatment of patients with ER-positive BC with eribulin after endocrine therapy plus CDK4/6 inhibitors.

\section{Materials and Methods}

Compounds. Eribulin mesylate (Halaven ${ }^{\circledR}$ ) was manufactured by Eisai Co., Ltd (Tokyo, Japan). Capecitabine, fulvestrant and palbociclib were purchased from AK Scientific (Union City, CA, USA), AstraZeneca (Cambridge, UK) and Selleck Chemicals (Houston, TX, USA), respectively.

Animal study. All protocols were approved by the Institutional Animal Care and Use Committee of Eisai Co., Ltd and implemented in accordance with the Animal Experimentation Regulations of Eisai Co., Ltd.

The 4 PDX models (OD-BRE-0188, -0438, -0450, -0745) were established by Oncodesign (Dijon, France) (13) and the IMODI consortium (14). To establish the PDX models, patient-derived tumor tissues were collected under informed consent and fresh tumor was subcutaneously implanted into Balb/C nu/nu mice (Charles River, Wilmington, MA, USA). After tumor development, the mice were euthanized and each tumor was excised, collected and cut into small fragments, which again were implanted singly into $\mathrm{Balb} / \mathrm{C}$ nu/nu mice. When each tumor had reached 500 to 1000 $\mathrm{mm}^{3}$, it was surgically excised, cut into small fragments (30 to 50 $\mathrm{mg}$ ) and frozen in RPMI1640 medium containing 10\% FCS and $10 \%$ DMSO for preservation. To induce patient-derived tumors using established models, preserved tumor fragments were thawed and subcutaneously implanted singly into SWISS-Nude mice (Charles River) or NOG mice (Taconic Biosciences, Rensselaer, NY, USA). When each tumor had reached 500 to $1500 \mathrm{~mm}^{3}$, it was surgically excised and cut into fragments ( 30 to $50 \mathrm{mg}$ ), which were subcutaneously implanted singly into SWISS-Nude mice (Charles River). When the tumor had reached 100 to $200 \mathrm{~mm}^{3}$, mice were randomized into each treatment group ( 2 to 4 mice per group) and treatment with fulvestrant, palbociclib, or fulvestrant plus palbociclib, or eribulin, or capecitabine was started (day 0 ) in each of the 4 PDX models. Fulvestrant was administered subcutaneously at $5 \mathrm{mg} /$ mouse (Q7D $\times 3$; every 7 days for 3 doses), and palbociclib was diluted with $0.9 \% \mathrm{NaCl}$ and administered by oral gavage at 100 $\mathrm{mg} / \mathrm{kg}(\mathrm{Q} 1 \mathrm{D} \times 21)$. Capecitabine was diluted with 5\% Arabic gum/ $40 \mathrm{mM}$ citrate buffer ( $\mathrm{pH}$ 6.0) and administered by oral gavage at around the maximum tolerated dose (MTD) $(250 \mathrm{mg} / \mathrm{kg})(\mathrm{Q} 1 \mathrm{D} \times 14)$. Eribulin was diluted with physiological saline solution and intravenously injected via the tail vein on a $\mathrm{Q} 4 \mathrm{D} \times 3$ at about $1 / 4$ MTD (0.5-0.57 mg/kg, Eri-L) and MTD (2.0-2.26 mg/kg, Eri-H) of eribulin (for OD-BRE-0188 and OD-BRE-0745 models; $0.5 \mathrm{mg} / \mathrm{kg}$ and $2.0 \mathrm{mg} / \mathrm{kg}$, for OD-BRE-0450 model; $0.57 \mathrm{mg} / \mathrm{kg}$ and $2.0-2.26$ $\mathrm{mg} / \mathrm{kg}$, for OD-BRE-0438 model; $0.5-0.57 \mathrm{mg} / \mathrm{kg}$ and $2.0-2.26$ $\mathrm{mg} / \mathrm{kg}$ ). Tumor size was measured twice each week, and the length and width of tumor was measured with caliper and tumor volume was estimated by the formula $\mathrm{V}=\left(\right.$ width $^{2} \times$ length $) / 2$. Relative tumor volume (RTV) compared with the initial tumor volume was also calculated.
To evaluate the progression-free survival of mice, we conducted Kaplan-Meier estimates of survival across the 4 PDX models. Progression-free survival duration was defined as the number of days $t$ until the tumor volume reached 4 times the initial tumor volume or dead or euthanasia because of ethical issues in accordance with the Animal Experimentation Regulation. Events in which the tumor did not reach 4 times the initial tumor volume were censored during experimental period (189 days for OD-BRE0188, 154 days for OD-BRE-0438, 164 days for OD-BRE-0450, and 222 days for OD-BRE-0745). The best overall response was determined by comparing the tumor volume change on day $t$ with the baseline volume on day $0(15)$. The formula $\Delta \mathrm{Vol}_{\mathrm{t}}=\left[\left(\mathrm{V}_{\mathrm{t}}\right.\right.$ $\left.\mathrm{V}_{\text {initial }} / \mathrm{V}_{\text {initial }}\right] \times 100 \%$ was used to calculate the percentage change from baseline, and the best overall response was the minimum value of $\Delta \mathrm{Vol}_{\mathrm{t}}$ for $t \geq$ day 21 . Data on mice that were euthanized because of ethical issues in accordance with the Animal Experimentation Regulation before completion of 21 days of treatment were removed from the data set.

Sequential treatments after fulvestrant plus palbociclib. To examine the effect of sequential treatments after fulvestrant plus palbociclib in the OD-BRE-0438 model, tumor fragments were subcutaneously implanted singly into NOD-SCID mice (NOD.CB17-Prkd $c^{\text {scid/J; }}$ Charles River). When the tumors had reached approximately 200 $\mathrm{mm}^{3}$ (day 0 ), mice were randomized into each treatment group to evaluate the antitumor activity of fulvestrant, palbociclib, and fulvestrant plus palbociclib for 14 days (6 mice per group), and fulvestrant plus palbociclib followed by the sequential treatments (74 mice). Fulvestrant was administered subcutaneously at 5 $\mathrm{mg} /$ mouse $(\mathrm{Q} 7 \mathrm{D} \times 2)$, and palbociclib was diluted with $50 \mathrm{mM} \mathrm{Na}-$ lactate buffer ( $\mathrm{pH} 4.0)$ and administered by oral gavage at 100 $\mathrm{mg} / \mathrm{kg}(\mathrm{Q} 1 \mathrm{D} \times 14)$. For immunohistochemical analysis, 14 days after combination treatment, mice were euthanized and the tumors were collected. To evaluate the antitumor activity of sequential treatments, 74 mice given the 14-day fulvestrant plus palbociclib regimen were re-randomized into each treatment group (6 mice per group), and further treatments with fulvestrant plus palbociclib, eribulin, or capecitabine were started (day 14). Fulvestrant was administered subcutaneously at $5 \mathrm{mg} /$ mouse $(\mathrm{Q} 7 \mathrm{D} \times 2)$, and palbociclib was diluted with $50 \mathrm{mM}$ Na-lactate buffer $(\mathrm{pH} 4.0)$ and administered by oral gavage at $100 \mathrm{mg} / \mathrm{kg}(\mathrm{Q} 1 \mathrm{D} \times 14)$. Eribulin was diluted with physiological saline solution and intravenously injected via the tail vein at 0.5 or $1.0 \mathrm{mg} / \mathrm{kg}(\mathrm{Q} 4 \mathrm{D} \times 3)$. Capecitabine was diluted with $5 \%$ Arabic gum/ $40 \mathrm{mM}$ citrate buffer ( $\mathrm{pH}$ 6.0) and administered by oral gavage at 125 or $250 \mathrm{mg} / \mathrm{kg}(\mathrm{Q} 1 \mathrm{D} \times 14)$. On day 28 after sequential treatments for 14 days, the mice were euthanized and the tumors were collected for immunohistochemical analysis. Tumor size was measured twice each week, and the RTV compared with the tumor volume on day 14 was also calculated for the sequential treatment-period.

Quantitative RT-PCR. When PDX tumor tissues in untreated SWISSNude mice became larger than $200 \mathrm{~mm}^{3}$, tumor tissues were excised and RNA was extracted and purified by using a Maxwell RSC simplyRNA Tissue Kit (Promega, Madison, WI, USA). One microgram of purified total RNA was reverse transcribed by using a High Capacity cDNA Reverse Transcription Kit (Thermo Fisher Scientific, Waltham, MA, USA). TaqMan Fast Advanced Master Mix (Thermo Fisher Scientific) and TaqMan Gene Expression Assay (Hs99999905_m1 for GAPDH, Hs01046816_m1 for ESR1, 


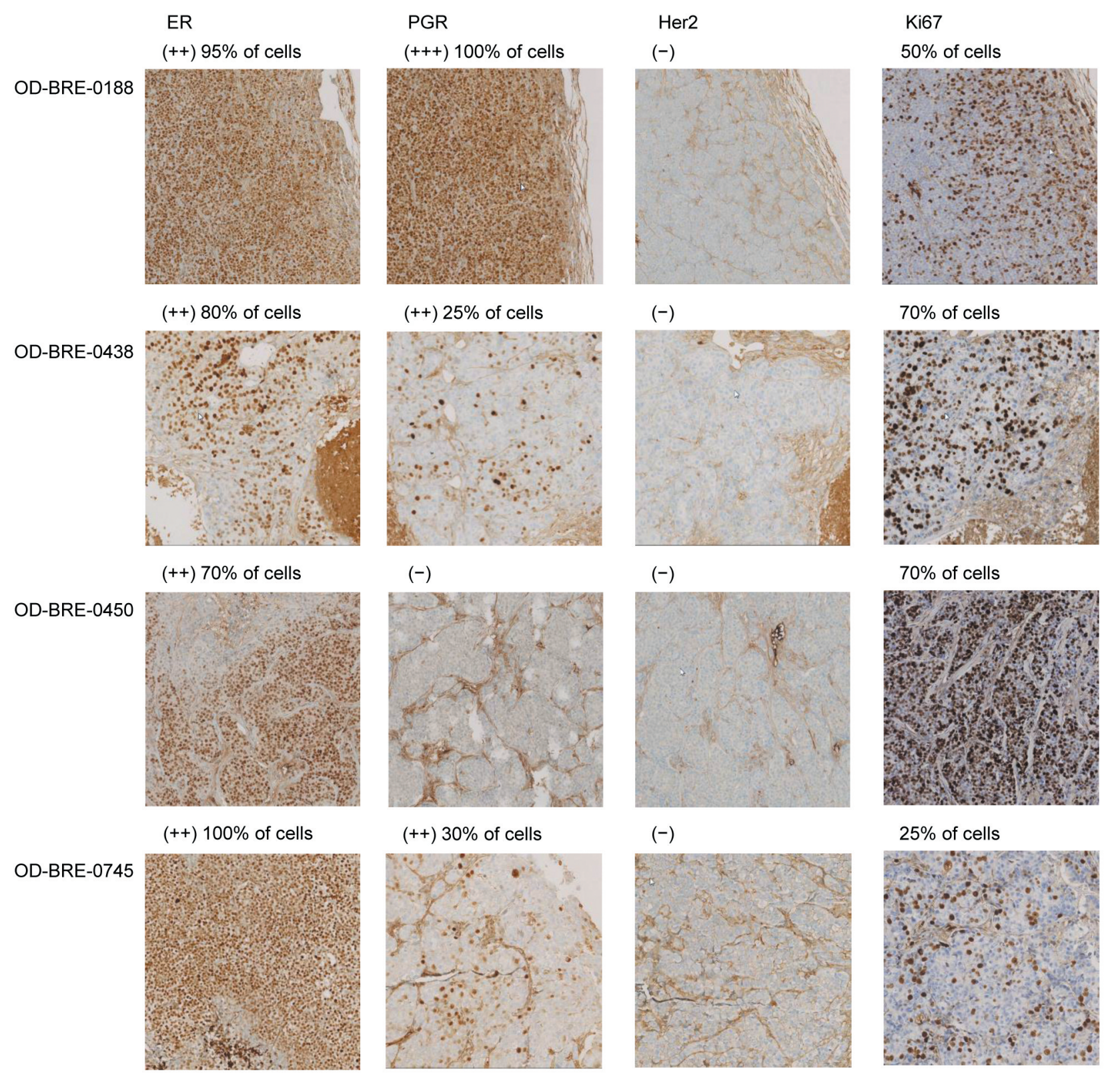

Figure 1. Subtypes of 4 luminal-type breast cancer patient-derived xenograft models. Representative immunohistochemical images show the expression of estrogen receptor (ER), progesterone receptor (PGR), human epithelial growth factor receptor 2 (Her2) and Ki67. Magnification, $\times 100$.

Hs01556702_m1 for progesterone receptor (PGR), Hs01001580_m1 for ERBB2, Hs01032443_m1 for MKI67, Hs01023895_m1 for CDH1, Hs00983056_m1 for CDH2, Hs00958111_m1 for VIM, Hs00232783_m1 for ZEB1; Thermo Fisher Scientific) were used for quantitative RT-PCR, which was performed on a QuantStudio 7 system (Thermo Fisher Scientific). Ct values for each sample were measured in duplicate and mean $\mathrm{Ct}$ values were used for analysis. Changes in gene expression were assessed by comparing the deltadelta $\mathrm{Ct}$ values for each sample, with GAPDH as a housekeeping gene.
RNA sequencing (RNA-Seq). Using tumor tissues collected from untreated mice $(n=4)$ embedded in O.C.T. compound, sections were sliced at $10 \mu \mathrm{m}$ thickness using a cryostat and RNA was purified using a Maxwell RSC simplyRNA Tissue Kit (Promega). Sequencing libraries were generated using SureSelect StrandSpecific RNA Library Prep (Agilent Technologies, Santa Clara, CA, USA). Paired-end sequencing of $2 \times 150 \mathrm{bp}$ was conducted with an Illumina NextSeq 500 system (Illumina, San Diego, CA, USA). Experiments were conducted in accordance with the manufacturer's instructions. FASTQ files for each sample converted from the 
Illumina BCL files were entered into FastQC (16) for quality assessment. Sequencing data were processed by using Trimmomatic (v0.36) (17) to remove all Illumina adaptor sequences and bases with low quality scores. Filtered reads were mapped to the human genome (GRCh38) and the mouse genome (mm10) using STAR (v2.5.2b) (18). Gene expression levels were estimated in transcripts per million (TPM) for all samples using RSEM (v1.2.31) (19). For molecular characterization of the PDX models, subtype marker genes of BC (ESR1, PGR, ERBB2 and MKI67) were selected to confirm the gene expression profiles in OD-BRE-0438 model.

Immunohistochemical analysis. For subtype analysis of the established 4 PDX models, tumors were fixed in 10\% formalin and embedded in paraffin. Immunohistochemical analysis was performed using the automatic VENTANA BenchMark XT system (Ventana Medical Systems, Oro Valley, AZ, USA). After a first step of deparaffinization and rehydration, tissue sections were incubated in Cell Conditioning Solution (CC1, Ventana Medical Systems) for antigen unmasking. Anti-HER2/neu (clone 4B5) rabbit monoclonal primary antibody (Ventana Medical Systems, $6 \mu \mathrm{g} / \mathrm{ml}$ final), antiKi-67 (clone MIB-1) mouse monoclonal primary antibody (Agilent Technologies, Santa Clara, CA, USA, dilution 1/50), anti-estrogen receptor (clone SP1) rabbit monoclonal primary antibody (Ventana Medical Systems, $1 \mu \mathrm{g} / \mathrm{ml}$ final), and anti-progesterone receptor (clone 1E2) rabbit monoclonal primary antibody (Ventana Medical Systems, $1 \mu \mathrm{g} / \mathrm{ml}$ final) were used for immunohistochemical analysis. After a washing step, counterstaining with hematoxylin was performed. Finally, dehydration and stabilization of section with mounting medium was performed before the quantification of specific antigen using microscope (magnification $\times 100$ ).

For immunohistochemical analysis of treated tumors, they were fixed in $10 \%$ formalin and embedded in paraffin. For ER staining, tissue sections were deparaffinized and treated with Dako REAL Target Retrieval Solution ( $\mathrm{pH}$ 6.0; Agilent Technologies) for $20 \mathrm{~min}$ at $98^{\circ} \mathrm{C}$. After endogenous peroxidase activity had been blocked by the addition of $3 \%$ hydrogen peroxide for $10 \mathrm{~min}$, samples were stained with anti-estrogen-receptor-alpha antibody (clone SP1, ABCAM, Cambridge, UK). SignalStain Boost IHC Detection Reagent (HRP, Rabbit; Cell Signaling Technologies, Danvers, MA, USA) was used as a secondary antibody; it was detected with diaminobenzidine and then counterstained with hematoxylin. Stained samples were scanned with an Aperio ScanScope XT system (Leica Biosystems, Wetzlar, Germany), and the whole tissue was analyzed by using the HALO image analysis platform (Indica Labs, Albuquerque, NM, USA) to quantify ER expression in tumor cells. First, cells in whole tissue were classified to select tumor cells. Expression levels of ER were classified as high $(3+)$, moderate $(2+)$, low $(1+)$ or negative $(0+)$, and the tumor cell number and percentage at each expression level were calculated. H-scores were determined by using the formula: $\mathrm{H}$-score $=3 \times(\%$ of $3+$ cells $)+2 \times(\%$ of $2+$ cells $)+1 \times(\%$ of $1+$ cells $)$.

For E-cadherin staining, tissue sections were deparaffinized and treated with an immunohistochemical antigen retrieval reagent (EDTA, pH 8.0; Enzo Life Sciences, Farmingdale, NY, USA) for 5 min at $121^{\circ} \mathrm{C}$. After endogenous peroxidase activity had been blocked by the addition of $3 \%$ hydrogen peroxide for $20 \mathrm{~min}$,

\section{A}
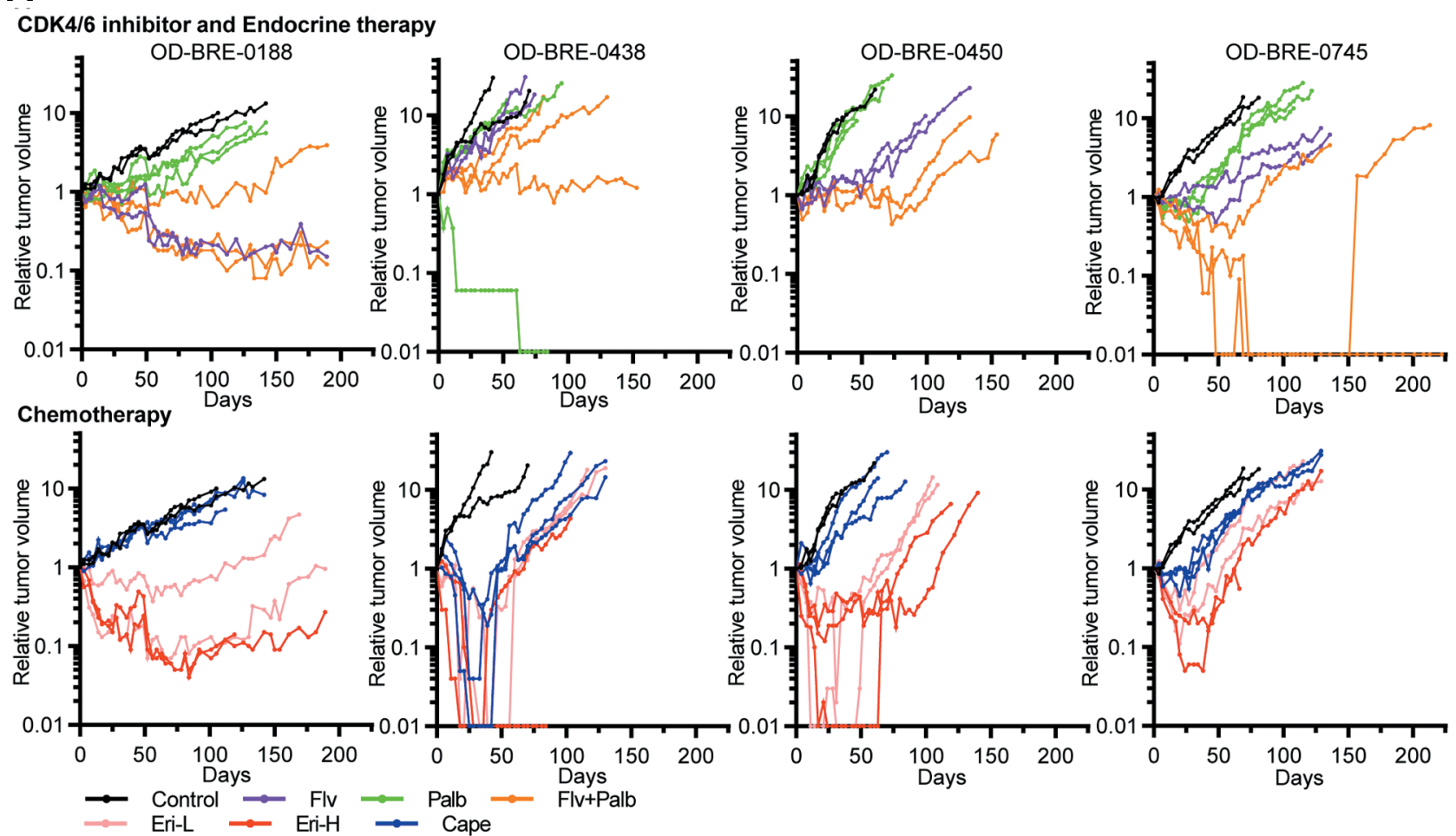

Figure 2. Continued 

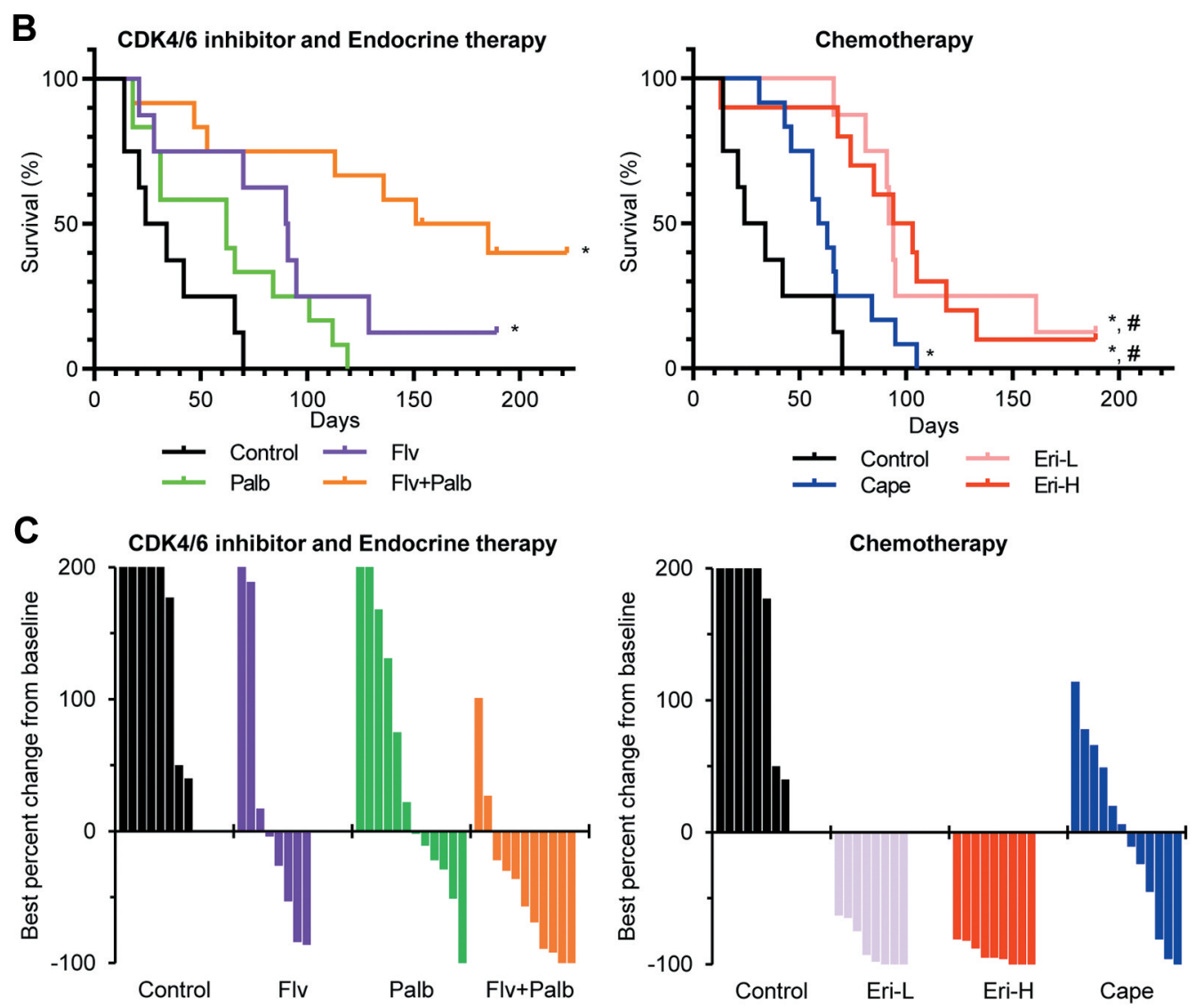

Figure 2. Antitumor activity of CDK4/6 inhibitor, endocrine therapy and chemotherapy in 4 luminal-type breast cancer patient-derived xenograft (PDX) models. PDX tumor-bearing mice were randomized into each treatment group ( $\mathrm{n}=2$ to 4 per group), and treated with $5 \mathrm{mg} / \mathrm{mouse}$ fulvestrant (Flv; purple) on a Q7D $3 ; 100 \mathrm{mg} / \mathrm{kg}$ palbociclib (Palb; green) on a Q1D 21 ; a combination of Flv plus Palb (orange); 0.5-0.57 mg/kg of eribulin (Eri-L; pink), and 2.0-2.26 mg/kg of eribulin (Eri-H; red) on a Q4D 3 ; or $250 \mathrm{mg} / \mathrm{kg}$ capecitabine (Cape; blue) on a Q1D $\times 14$. Untreated control is shown in black. The same control group data are shown in the upper (CDK4/6 inhibitor and endocrine therapy) and lower (chemotherapy) panels. (A) Relative tumor volumes are shown for days after treatment initiation. Minimum relative tumor volumes (RTVs) for individual mice, which were lower than 0.01 are shown as 0.01. (B) Survival of mice. Kaplan-Meier estimates of mouse survival combined across the 4 PDX models are shown ( $n=8$ to 12 per treatment group). Mouse survival was defined as the number of days $t$ until the tumor volume reached four times the initial tumor volume. Differences in survival were examined by using the log-rank (Mantel-Cox) test. ${ }^{*} p<0.05$ vs. Control by log-rank test. ${ }^{\#} p<0.05$ vs. Cape by log-rank test. (C) Waterfall plots. The best overall response as percentages from baseline across the 4 PDX models are shown. The best overall response was determined by comparing the tumor volume change at day $t$ with the baseline tumor volume at day 0 for $t \geq 21$ (after end of treatment period). Maximum values of best percentage change from baseline are shown as $200 \%$. Best percentage changes from baseline that exceeded $200 \%$ are represented as 200\%. The same control data are used in the left (CDK4/6 inhibitor and endocrine therapy) and right (chemotherapy) panels.

samples were stained with anti-E-cadherin antibody (clone 24E10, Cell Signaling Technologies). Histofine Simple Stain MAX PO (R) (Nichirei Biosciences, Tokyo, Japan) was used as a secondary antibody; it was detected with diaminobenzidine and then counterstained with hematoxylin. Quantification of E-cadherin expression on membranes was conducted by GeneticLab (Sapporo, Japan). Stained samples were scanned with a ScanScope AT Turbo (Leica Biosystems), and the whole tissue was analyzed by the Aperio GENIE histomorphology image analysis tool (Leica Biosystems) to remove stromal cells and select only tumor cells for subsequent analysis. After classification of tumor cells, E-cadherin expression on the membranes of tumor cells was quantified by using Aperio Membrane Algorithm v9 (Leica Biosystems) and classified into expression levels of high (3+), moderate (2+), low (1+) and negative $(0+)$, and the cell numbers and the percentage of each expression-level population in tumor cells were calculated. H-scores were determined by using the formula described above.

Statistical analyses. Statistical analyses were performed with GraphPad Prism v8.3.1 software (GraphPad Prism Software, Inc., San Diego, CA, USA). An unpaired $t$-test, one-way ANOVA with Dunnett's multiple comparisons test and two-way ANOVA with Sidak's multiple comparisons test were performed to confirm statistical significance. Differences in survival were examined by using a log-rank (Mantel-Cox) test. $p$-Values $<0.05$ were considered statistically significant. 
Table I. RNA-Seq analysis of gene expression levels in the OD-BRE-0438 model.

\begin{tabular}{lrrrrrrrr}
\hline Gene & ESR1 & PGR & ERBB2 & MKI67 & CDH1 & CDH2 & VIM & ZEB1 \\
\hline TPM & 246.9 & 11.8 & 42.3 & 41.1 & 330.2 & 0.8 & 196.4 & 0.1 \\
SEM & 19.3 & 3.0 & 1.2 & 3.1 & 25.2 & 0.2 & 28.2 & 0.1 \\
\hline
\end{tabular}

TPM: Transcripts per million; SEM: standard error of the mean.

\section{Results}

Antitumor activity of endocrine therapy, CDK4/6 inhibitor, and chemotherapy in 4 luminal-type BC PDX models. We first determined the antitumor activity in 4 established luminal-type BC PDX models, consisting of one luminal Atype model (OD-BRE-0188) and three luminal B-type models (OD-BRE-0438, OD-BRE-0450 and OD-BRE-0745). The subtypes of these $\mathrm{BCs}$ were confirmed by immunohistochemical staining for ER, PGR, human epithelial growth factor receptor 2 (Her2) and Ki67 (Figure 1 ). We examined the antitumor activity of fulvestrant and palbociclib monotherapies, and of fulvestrant plus palbociclib combination therapy, in these 4 PDX models (Figure 2A upper panels, Figure 2B left panel, Figure 2C left panel). Fulvestrant clearly inhibited in vivo tumor growth in 3 of the 4 PDX models examined, and there were some mice in the OD-BRE-0188 and -0745 models in which the tumors shrank. The OD-BRE-0438 model seemed to be resistant to fulvestrant. Palbociclib had antitumor activity in 2 out of the 4 PDX models by slowing tumor growth. Fulvestrant plus palbociclib combination therapy showed clear antitumor activity in all 4 PDX models and resulted in tumor shrinkage in OD-BRE-0188 and OD-BRE-0745 models. Among the endocrine-based therapies, fulvestrant plus palbociclib combination therapy gave the most robust survival, followed by fulvestrant; these survival rates were significantly greater than in the controls (Figure 2B left panel, Flv vs. Control: $p=0.0063$, Flv+Palb vs. Control: $p=0.0003)$. In separate studies, eribulin was intravenously injected via the tail vein at $2.0 \mathrm{mg} / \mathrm{kg}(\mathrm{Q} 4 \mathrm{D} \times 3)$, and capecitabine was administered by oral gavage at $500 \mathrm{mg} / \mathrm{kg}(\mathrm{Q} 1 \mathrm{D} \times 14)$. In this experiment, one mouse treated with capecitabine at $500 \mathrm{mg} / \mathrm{kg}$ showed a remarkable reduction in body weight (data not shown). Thus, this dose was judged to be intolerable and we determined that the maximum tolerated dose (MTD) of eribulin under a $\mathrm{Q} 4 \mathrm{D} \times 3$ was about $2.0 \mathrm{mg} / \mathrm{kg}$ and that of capecitabine under a Q1D $\times 14$ was about $250 \mathrm{mg} / \mathrm{kg}$ in this model. In our analysis of the antitumor activity of chemotherapy in the 4 PDX models, capecitabine at MTD $(250 \mathrm{mg} / \mathrm{kg})$ showed clear antitumor activity against 3 of the 4 PDX models, the exception being OD-BRE-0188. Eribulin at either dose (1/4 MTD of Eri (Eri-L) or MTD of Eri (Eri-H)) had antitumor activity in all 4 models (Figure 2A lower panels). However, treatment with capecitabine resulted in tumor shrinkage only in the OD-BRE-0438 model, whereas eribulin treatment at either dose resulted in tumor regression in all 4 models (Figure 2A lower panels). Compared with the controls, eribulin and capecitabine both significantly promoted survival in all 4 PDX models (Figure $2 \mathrm{~B}$ right panel, Eri-L vs. Control: $p=0.0001$, Eri-H vs. Control: $p=0.0004$, Cape vs. Control: $p=0.035)$, and eribulin at either doses gave significantly superior survival compared to capecitabine (EriL vs. Cape: $p=0.0229$, Eri-H vs. Cape: $p=0.0093)$. A waterfall plot describing the best overall response percentage from baseline across the 4 PDX models also demonstrated that fulvestrant plus palbociclib combination had most robust tumor response in endocrine-based therapy (Figure 2C left panel), and eribulin at either dose gave a tumor response superior to that given by capecitabine (Figure $2 \mathrm{C}$ right panel). These results suggested that eribulin had more potent antitumor activity than capecitabine in the 4 luminal-type BC PDX models.

Molecular characterization of the 4 luminal-type BC PDX models. The sensitivity of the OD-BRE-0438 model to the fulvestrant plus palbociclib combination was the most modest among the 4 PDX models (Figure 2A upper panels), suggesting that this model was relatively resistant to fulvestrant-palbociclib combination therapy. In contrast, the OD-BRE-0438 model was the most sensitive to both eribulin and capecitabine. We conducted an RNA-Seq analysis in the OD-BRE-0438 model to characterize the molecular profiles of this model and evaluate the mRNA expression levels of the hormone receptors ESR1, PGR and ERBB2, the proliferation marker MKI67 and the EMT markers CDH1, CDH2, VIM and ZEB1 (Table I). Representative EMT marker genes ( $C D H 1, C D H 2, V I M$ and $Z E B 1)$ were selected for molecular characterization of the PDX model, because eribulin reverses EMT activity (11). We plotted the levels of expression of these genes in each model relative to the ODBRE-0438 model (Figure 3). All 4 PDX models expressed ESR1, and OD-BRE-0188 expressed high levels of PGR. Among the EMT marker genes, $C D H 1$ expression was detected in three luminal B-type models OD-BRE-0438, ODBRE-0450 and OD-BRE-0745 but not in the OD-BRE-0188 

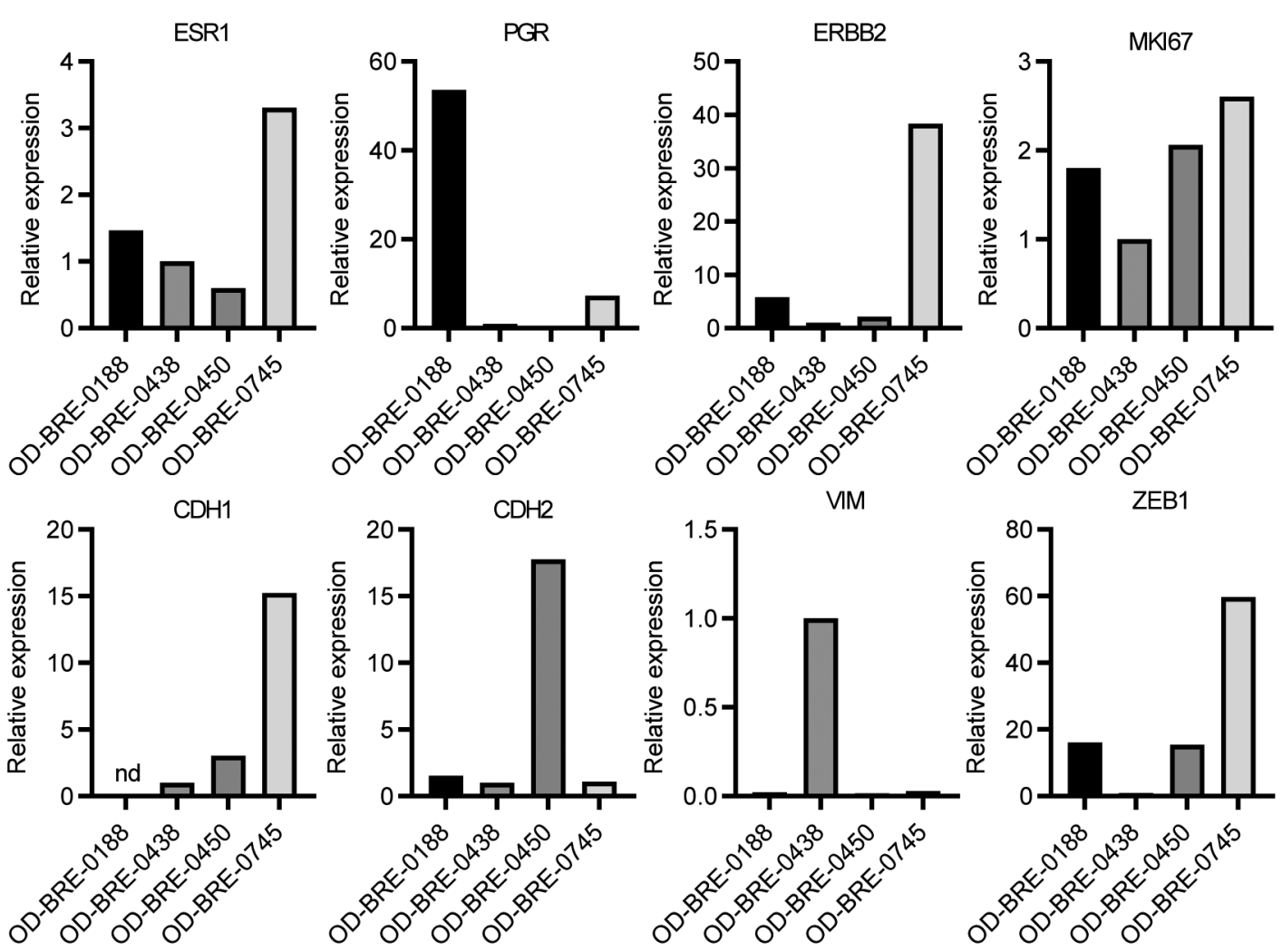

Figure 3. Molecular profiles of the 4 luminal-type breast-cancer patient-derived xenograft (PDX) models. RNA was extracted and purified from resected tumor tissues from untreated mice ( $n=1$ per model). After synthesis of cDNA, quantitative RT-PCR was performed, and mRNA expression levels of genes relative to ones in the OD-BRE-0438 model were calculated. Ct values for each sample were measured in duplicate and mean Ct values were used for analysis. nd: Not detected.

model (Figure 3). Furthermore, OD-BRE-0438 expressed characteristically high levels of VIM (Table I and Figure 3). Although the relative expression levels of $C D H 2$ and $Z E B 1$ were lower in the OD-BRE-0438 model than in the other models (Figure 3), their expression levels were quite low in OD-BRE-0438 (Table I), and these differences may not be meaningful. Based on the sensitivities to drug treatments and the molecular profiles of the PDX models (low $\mathrm{CDH} 1$ and high VIM expression), we selected the OD-BRE-0438 model for further analysis of the antitumor activities of eribulin and capecitabine after fulvestrant plus palbociclib therapy.

Evaluation of the antitumor activity of chemotherapy in the OD-BRE-0438 model after fulvestrant plus palbociclib therapy. In the OD-BRE-0438 model, we compared the antitumor activity of fulvestrant, palbociclib, and fulvestrant plus palbociclib combination therapy for 2 weeks (day 0-day 13) with the control (no treatment), and that for 4 weeks compared with the $2^{\text {nd }}$ control (day 0-day 13 with fulvestrant plus palbociclib, and day 14-day 27 with no-treatment) (Figure
4A to C). In the first experiment, we treated mice with fulvestrant, palbociclib, or fulvestrant plus palbociclib for 2 weeks. In the second experiment, after 2 weeks of treatment with the fulvestrant plus palbociclib combination, mice were divided into two groups. One group continued to receive treatment with fulvestrant plus palbociclib and the other group received no further treatment (Figure 4A). In the first experiment, treatments for 2 weeks with fulvestrant, palbociclib, or fulvestrant plus palbociclib had significant antitumor activity compared to controls (Figure 4B, Flv vs. Control: $p=0.0031$, Palb vs. Control: $p<0.0001$, Flv+Palb vs. Control: $p<0.0001)$, and only treatments with fulvestrant plus palbociclib led to tumor dormancy (Figure 4B). In the second experiment, treatments with fulvestrant plus palbociclib kept tumor dormancy, but no tumor shrinkages were observed. However, in the group that received no further treatment from days 14 to 28 , the tumors started to grow again (Figure 4C).

Immunohistological analysis using anti-ER antibody demonstrated that 2 weeks of treatment with fulvestrant plus palbociclib significantly decreased ER expression compared 


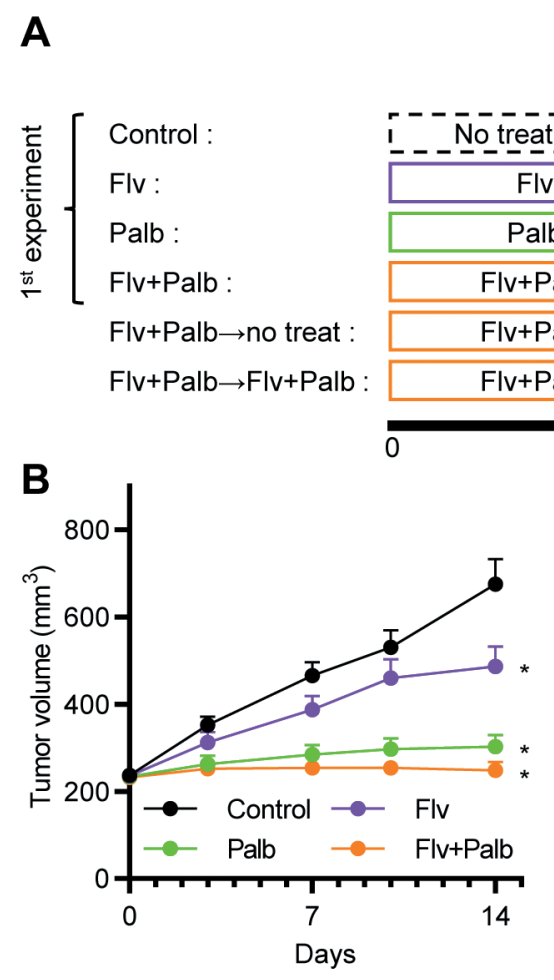

with the controls in the first experiment. In the second experiment, ER expression was restored in the non-treated group as we expected with the washout of drugs. However, despite of continued treatment with fulvestrant plus palbociclib, ER expression was restored after the 2 weeks of combination treatments compared with the decreased ER expression after the first 2-weeks combination treatment (Figure 4D and E), even though the antitumor activity of fulvestrant plus palbociclib was maintained. These results suggest that the resistance of ODBRE-0438 tumors to fulvestrant plus palbociclib developed during the 4 weeks of treatment.

We also performed immunohistochemical staining to qualitatively and quantitatively evaluate E-cadherin expression on the membranes of tumor cells (Figure 4F and G). Two weeks of treatment with fulvestrant plus palbociclib significantly increased E-cadherin expression on cell membranes compared with that in the controls (Figure 4G). This increased expression of E-cadherin did not change further with fulvestrant plus palbociclib treatment, and interestingly in the absence of further treatment for an additional 2 weeks, those changes were maintained (Figure 4G).

Based on these observations, we next administered sequential treatments with fulvestrant plus palbociclib for 2 weeks, followed by either eribulin or capecitabine for a further 2 weeks to determine the antitumor activity of chemotherapy after CDK4/6 inhibitor plus endocrine therapy. The
Euthanasia

(day14)

$-\mathbf{A}^{-1}$

-

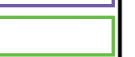

$2^{\text {nd }}$ experiment Euthanasia

lalb
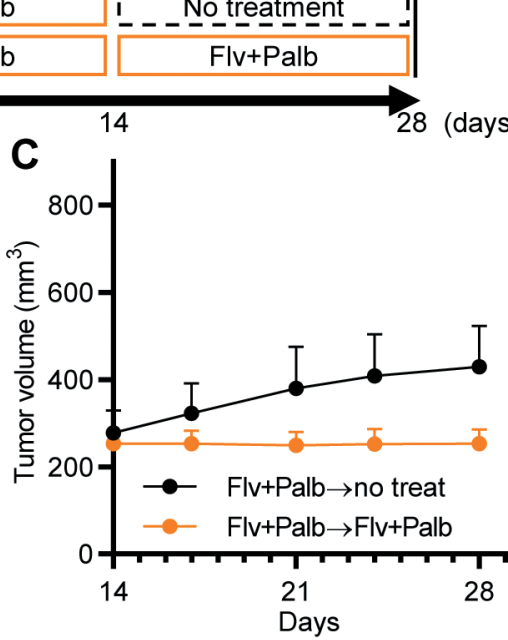

Figure 4. Continued

experimental design of these sequential treatments is shown in Figure 5A. After 2 week-treatments with fulvestrant plus palbociclib, treated mice were divided into 4 groups in the ODBRE-0438 model. We switched fulvestrant plus palbociclib to either eribulin $(0.5$ or $1.0 \mathrm{mg} / \mathrm{kg}$ ) or capecitabine (125 or 250 $\mathrm{mg} / \mathrm{kg}$ ), which was then given for an additional 2 weeks (Figure 5A). Treatment with either of these drugs from days 14 to 28 resulted in tumor regression without body weight loss (Figure 5B). Furthermore, we found that eribulin had significantly stronger antitumor activity than capecitabine at both the lower and the higher doses of each (Figure 5C).

Next, we explored the effects of eribulin or capecitabine on the expression of ER and E-cadherin. The expression levels of ER following eribulin or capecitabine treatment (at either the lower or the higher doses) after fulvestrant plus palbociclib did not differ (Figure 5D and E). Next, we performed immunohistochemical staining using anti-E-cadherin antibody to evaluate the effects of eribulin or capecitabine on E-cadherin expression on the cell membranes in the OD-BRE-0438 model (Figure 5F). At both the lower and higher doses of the two drugs, membrane E-cadherin expression was significantly higher in eribulin-treated tumors than that in capecitabinetreated tumors (Figure 5G, Eri $0.5 \mathrm{mg} / \mathrm{kg}$ vs. Cape $125 \mathrm{mg} / \mathrm{kg}$ : $p=0.0213$, Eri $1.0 \mathrm{mg} / \mathrm{kg} v s$. Cape $250 \mathrm{mg} / \mathrm{kg}$ : $p=0.0002)$. Thus, we clearly demonstrated the difference between eribulin and capecitabine for E-cadherin expression on the membrane. 
D

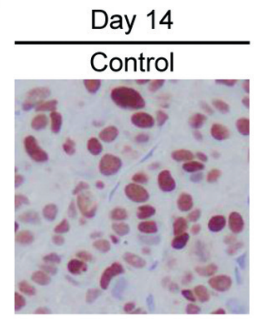

Flv+Palb
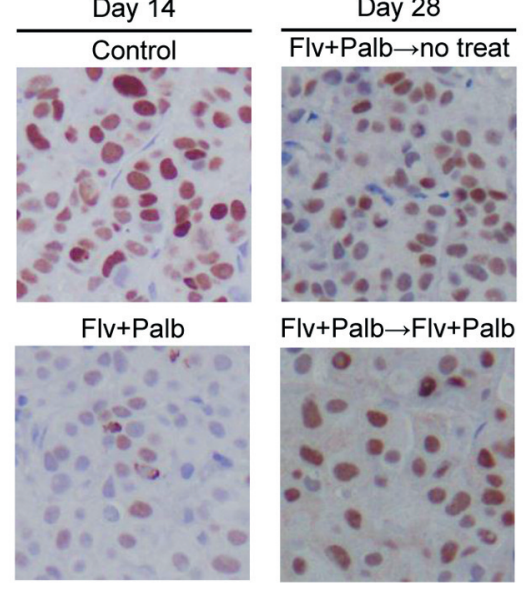

$\mathrm{Flv}+\mathrm{Palb} \rightarrow \mathrm{Flv}+\mathrm{Palb}$

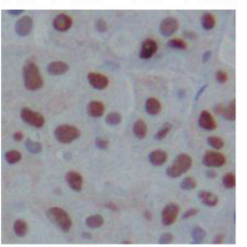

F

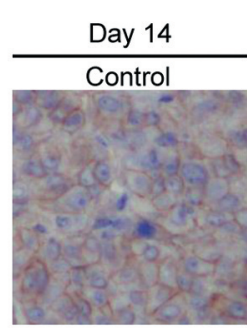

Flv+Palb

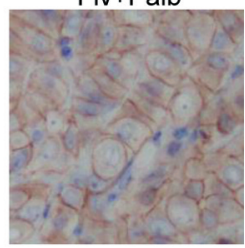

E

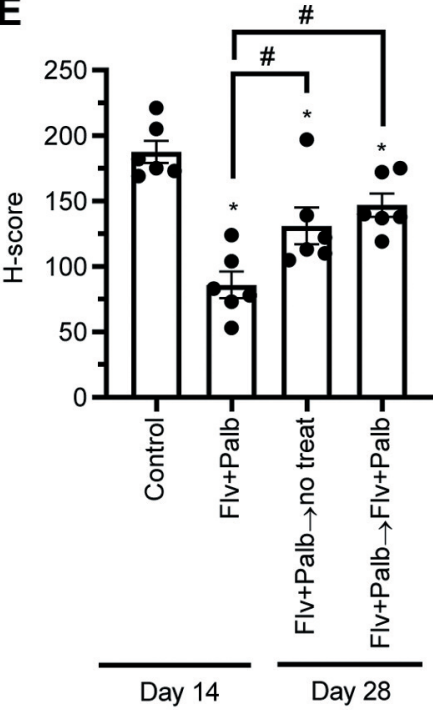

G

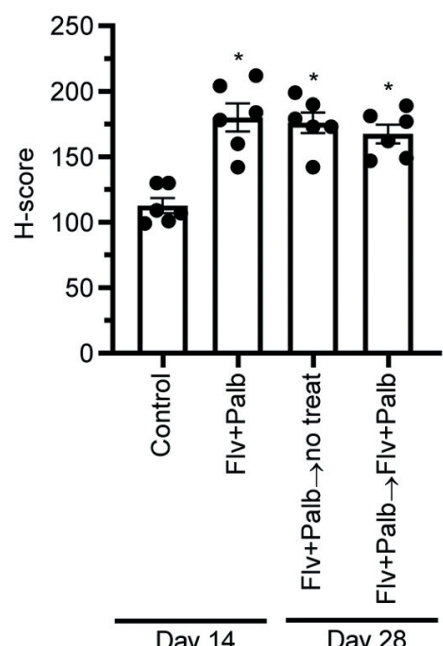

Figure 4. Antitumor activity of endocrine therapy plus CDK4/6 inhibitor combination treatment in the OD-BRE-0438 model. (A) Scheme of treatment with fulvestrant (Flv), palbociclib (Palb), or Flv plus Palb in the $1^{\text {st }}$ and $2^{\text {nd }}$ experiments. (B) Antitumor activity of Flv, Palb, or Flv plus Palb combined for an initial 2 weeks, days 0 and 14 (1 $1^{\text {st }}$ experiment). OD-BRE-0438 tumor-bearing mice were treated with Flv (purple, $\left.5 \mathrm{mg} / \mathrm{mouse}, Q 7 D \times 2\right)$, palbociclib (green, $100 \mathrm{mg} / \mathrm{kg}, Q 1 D \times 14$ ), or their combination (orange). Untreated control is shown in black. $n=6$ per group. Data shown are means $+S E M . * p<0.05$ vs. control by repeated measures Dunnett's multiple comparisons test using log-transformed values. (C) Antitumor activity of Flv plus Palb for 2 weeks, days 14 and 28 ( $2^{\text {nd }}$ experiment). Mice treated with Flv plus Palb for 2 weeks were randomized into two groups ( $n=6$ per group). One group continued treatment with Flv plus Palb (Flv + Palb $\rightarrow$ Flv + Palb, orange) and the other group received no further treatment $(F l v+$ Palb $\rightarrow$ no treat, black). Data shown are means $+S E M .(D-G)$ Tumor samples were collected on day 14 (untreated (control) or Flv+Palb) or on day $28(F l v+P a l b \rightarrow F l v+P a l b$ or Flv + Palb $\rightarrow n o$ treat). Representative immunohistochemical images of estrogen receptor $(E R)(D)$ and E-cadherin $(F)$ expression, and quantification of ER in tumor cells $(E)$ and E-cadherin on cell membranes of tumor cells $(G)$. ER expression in tumor cells $(E)$ or E-cadherin expression on membranes of tumor cells $(G)$ was classified into levels that were high (3+), moderate $(2+)$, low $(1+)$ or negative $(0+)$ and $H$-scores were calculated. $n=6$ per group. Data shown are means \pm SEM. ${ }^{*} p<0.05 \mathrm{vs}$. Control by Dunnett's multiple comparisons test. ${ }^{\#} p<0.05 \mathrm{vs}$. Flv + Palb by Dunnett's multiple comparisons test.

\section{Discussion}

Here, we showed the antitumor activity of endocrine therapy, CDK4/6 inhibitor, and chemotherapy in 4 luminal-type BC PDX models. In treatment-naïve mice, chemotherapy with eribulin caused tumor regression and had superior antitumor activity to capecitabine in the 4 PDX models (Figure 2). In the OD-BRE-0438 PDX model, which was relatively resistant to endocrine therapy, eribulin after endocrine therapy plus a CDK4/6 inhibitor had superior antitumor 


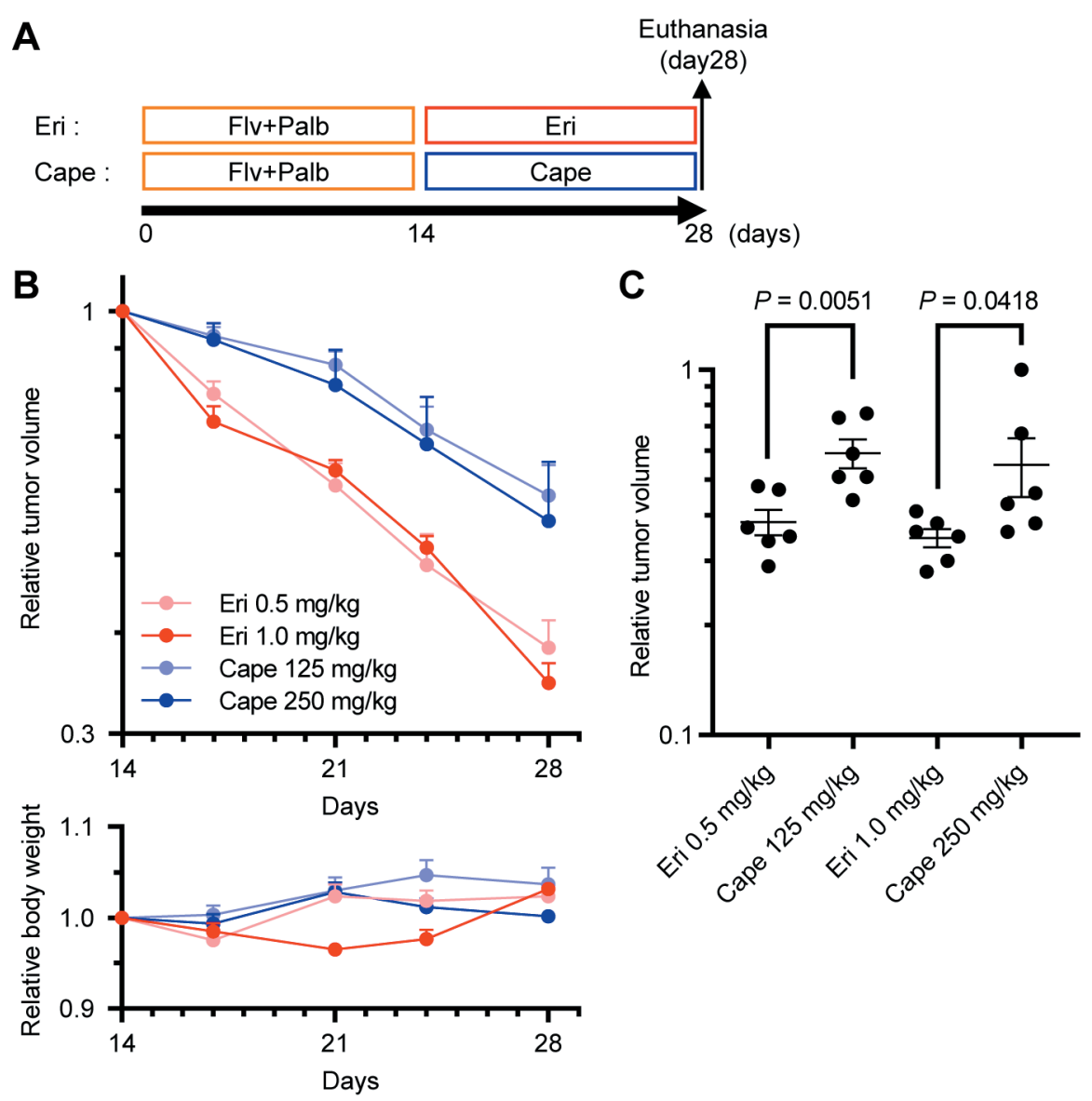

Figure 5. Continued

activity to capecitabine, as well as greater activity to increase E-cadherin expression.

With endocrine and CDK4/6 therapy in the 4 luminal-type PDX models, we demonstrated that fulvestrant plus palbociclib gave the most robust survival, followed by fulvestrant (Figure 2B). Clinically, CDK4/6 inhibitors are approved for use in addition to endocrine therapy, because CDK4/6 monotherapy has limited activity. In our 4 PDX models, we also observed that palbociclib monotherapy had limited antitumor activity, whereas fulvestrant plus palbociclib had improved antitumor activity compared with monotherapy (Figure 2), suggesting that these PDX models partly reflect the clinical phenotypes of luminal-type BC.

In the OD-BRE-0438 model under treatment-naïve conditions, the antitumor activities of eribulin and capecitabine were similar (Figure 2A). After fulvestrant plus palbociclib treatments, the antitumor activity of eribulin might not have been influenced by the prior CDK4/6 inhibitor treatment. But that of capecitabine seemed to have been attenuated, and eribulin had superior antitumor activity (Figure 5B and C). In this study, we used $250 \mathrm{mg} / \mathrm{kg}$ as MTD of capecitabine in this model because remarkable body weight loss was observed in one mouse treated with 500 $\mathrm{mg} / \mathrm{kg}$ capecitabine and this dose was judged to be intolerable in this model (data not shown). However, generally 500 $\mathrm{mg} / \mathrm{kg}$ capecitabine has been used as MTD in preclinical study (13). Thus, we used $1.0 \mathrm{mg} / \mathrm{kg}$ eribulin, which is half of MTD, as the higher dose for comparison with $250 \mathrm{mg} / \mathrm{kg}$ capecitabine and demonstrated superior antitumor activity of eribulin compared with capecitabine (Figure 5B and C). Recently, real-world data on the clinical outcomes of therapy with eribulin after CDK4/6 inhibitors has been reported (20). In that report, although longer term follow-up data are required for confirmation, the objective response rate (ORR) of patients with prior CDK4/6 inhibitor therapy who received eribulin treatment as per the US label was more than double the ORR in a pivotal phase III study of eribulin, and rates of selected adverse events did not exceed those observed in the EMBRACE trial (20). The results in that report were consistent with those in this preclinical study. Our preclinical results, together with real world data, might provide important preclinical and clinical evidence for eribulin 


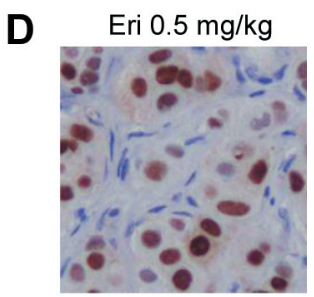

Cape $125 \mathrm{mg} / \mathrm{kg}$

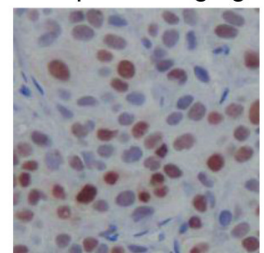

$\mathbf{F}$

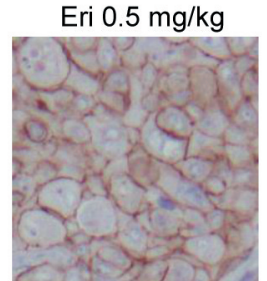

Cape $125 \mathrm{mg} / \mathrm{kg}$

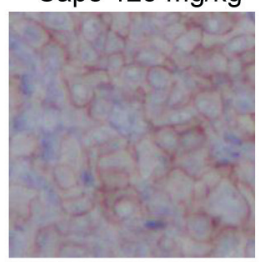

Eri $1.0 \mathrm{mg} / \mathrm{kg}$

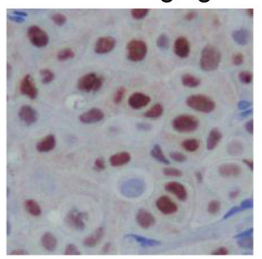

Cape $250 \mathrm{mg} / \mathrm{kg}$

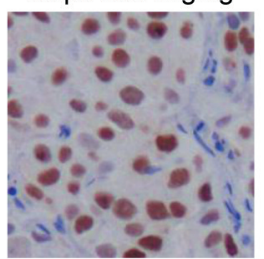

Eri $1.0 \mathrm{mg} / \mathrm{kg}$

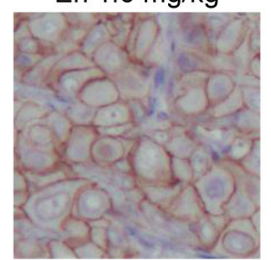

Cape 250 mg/kg
$\mathbf{E}$
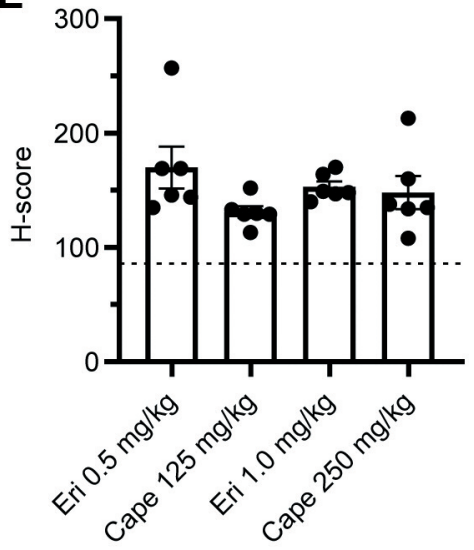

G

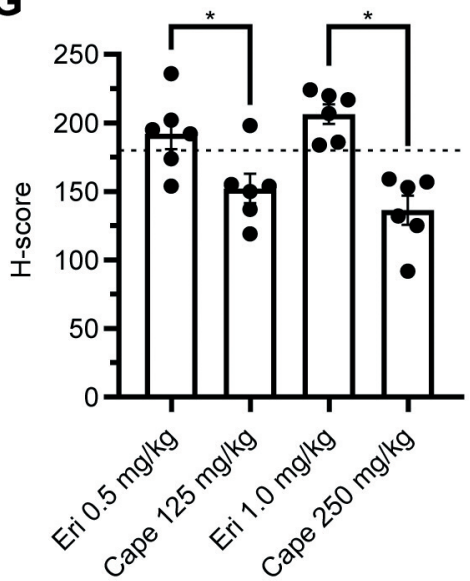

Figure 5. Antitumor activity of chemotherapy after endocrine therapy plus CDK4/6 inhibitor combination treatment in the OD-BRE-0438 model. (A) Scheme of sequential treatment with eribulin (Eri) and capecitabine (Cape) after fulvestrant (Flv) plus palbociclib (Palb) treatment. Flv plus Palb combination therapy was conducted for 2 weeks between days 0 and 14, and mice treated with Flv plus Palb for 2 weeks were randomized into two groups ( $n=6$ per group, day 14) and treated with either Eri or Cape for an additional 2 weeks. (B) Antitumor activity of Eri and Cape after Flv plus Palb combination treatment. Relative tumor volume (upper) and relative body weight (lower) compared with tumor volume and body weight on day 14 (after Flv+Palb therapy for 14 days and before chemotherapy treatments) from days 14 to 28 (chemotherapy period) are shown. Data shown are means + SEM. (C) Comparison of antitumor activity of Eri and Cape (day 28). Data shown are means \pm SEM. Statistical analysis was conducted by unpaired t-test using log-transformed values. (D-G) Tumor samples were collected on day 28 (initial 2 weeks of treatment with Flv plus Palb, followed by an additional 2 weeks of treatment with Eri or Cape, each at two doses. Representative immunohistochemical images of estrogen receptor $(E R)(D)$ and E-cadherin $(F)$ expression, and quantification of ER in tumor cells $(E)$ and E-cadherin on cell membranes of tumor cells $(G)$. ER expression in tumor cells $(E)$ or E-cadherin expression on membranes of tumor cells $(G)$ was classified into levels of high $(3+)$, moderate $(2+)$, low $(1+)$ and negative $(0+)$ and $H$-scores were calculated. $n=6$ per group. Data shown are means $\pm S E M$. Dotted lines in graphs indicate mean H-scores of Flv+Palb (day 14). ${ }^{*} p<0.05$ by two-way ANOVA with Sidak's multiple comparisons test.

treatment of patients with ER-positive $\mathrm{BC}$ who are resistant to endocrine therapy plus CDK4/6 inhibitors.

We conducted animal experiments to evaluate the antitumor activity of chemotherapy after 2 weeks of fulvestrant plus palbociclib treatments. Immunohistochemical analysis of ER expression in the OD-BRE-0438 model showed that 2 weeks of treatment with fulvestrant plus palbociclib suppressed ER expression, but an additional 2 weeks of treatment with the same combination did not maintain this effect on ER expression, and ER expression increased (Figure 4D and E). This suggests that resistance to fulvestrant plus palbociclib in this model occurred after 2 to 4 weeks of treatment. We also confirmed that treatment with fulvestrant plus palbociclib induced an increase in E-cadherin expression on the tumor cell membrane, and these effects of the combination treatment on E-cadherin were maintained for 4 weeks, even when the combination therapy was stopped at 2 weeks (Figure 4F and G). Previous reports have demonstrated that CDK4/6 inhibitors downregulate the EMT process (21-24). Mechanistically, the CDK4/6-DUB3- 
SNAIL1 and the CDK4/6-USP51-ZEB1 axis have been reported to induce EMT $(22,24)$. However, OD-BRE-0438 tumors poorly express SNAIL1 (TPM=1.3 by RNA-Seq) and ZEB1 (Table I) at baseline levels. Therefore, the increased expression of E-cadherin on the tumor cell membrane in response to fulvestrant plus palbociclib in the OD-BRE-0438 model might be independent of these reported pathways.

In this study, we demonstrated that eribulin-treated tumors express significantly higher levels of E-cadherin on the cell membrane compared with capecitabine-treated tumors after fulvestrant plus palbociclib (Figure 5G). In addition, cell membrane E-cadherin expression in tumors treated with capecitabine after fulvestrant plus palbociclib was significantly lower than that in non-treated tumors for 14 days after fulvestrant plus palbociclib (Cape $250 \mathrm{mg} / \mathrm{kg}$ in Figure $5 \mathrm{G} v s$. $\mathrm{Flv}+\mathrm{Palb} \rightarrow$ no treat in Figure $4 \mathrm{G}$, mean $\mathrm{H}$-score \pm SEM: $136.3 \pm 10.5$ vs. $176.0 \pm 8.0, p=0.0133$ by unpaired $t$-test). However, E-cadherin expression on the cell membranes of tumors treated with $1.0 \mathrm{mg} / \mathrm{kg}$ eribulin (Figure 5G) was significantly greater than that in the non-treated tumors for 14 days after fulvestrant plus palbociclib (Eri $1.0 \mathrm{mg} / \mathrm{kg}$ in Figure $5 \mathrm{G} v s$. Flv + Palb $\rightarrow$ no treat in Figure 4G, mean H-score \pm SEM: $206.3 \pm 7.1$ vs. 176.0 $\pm 8.0, p=0.0177$ by unpaired $t$-test). Furthermore, it was also significantly higher than that with fulvestrant plus palbociclib for a full of 28 days (Eri $1.0 \mathrm{mg} / \mathrm{kg}$ in Figure 5G vs. Flv+Palb $\rightarrow \mathrm{Flv}+\mathrm{Palb}$ in Figure 4G, mean $\mathrm{H}-$ score \pm SEM: $206.3 \pm 7.1$ vs. $167.5 \pm 7.1, p=0.0032$ by unpaired $t$-test), suggesting that treatment with eribulin appeared to increase E-cadherin expression on the cell membranes in the OD-BRE-0438 model compared with even fulvestrant plus palbociclib therapy. These results suggested that capecitabine treatment might induce EMT, demonstrating in this regard a clear difference from eribulin. Eribulin reversed EMT activity in tumor tissues after fulvestrant plus palbociclib therapy in the OD-BRE-0438 model, but capecitabine had the opposite activity. We found that capecitabine treatment decreased Ecadherin expression on the tumor cell membrane, which was increased after fulvestrant plus palbociclib treatments for 2 weeks, in the OD-BRE-0438 model (Figure 5G), suggesting that EMT was induced by capecitabine treatment. Resistance to fluorouracil - an active metabolite of capecitabine - is associated with a decrease in E-cadherin expression and an increase in the expression of mesenchymal markers in vitro $(25,26) . S-1$, an oral fluorouracil prodrug, has also been shown to decrease E-cadherin expression and increases vimentin expression in an in vivo xenograft model (26); this is consistent with our results. However, after fulvestrant plus palbociclib, eribulin induced E-cadherin expression on the cell membranes of OD-BRE-0438 tumors. Previous in vitro studies have shown that eribulin treatment increased E-cadherin expression and decreased $\mathrm{N}$-cadherin and vimentin expression at the mRNA and protein levels $(11,27)$. Furthermore, in an MX-1 BC in vivo xenograft model, reversal of EMT activity by eribulin has been confirmed (11). In clinical samples, a significant increase in E-cadherin expression has been observed in eribulin responders (28). Thus, reversal of EMT activity by eribulin has been demonstrated not only in preclinical experiments, but also in cancer patients. It is believed that the EMT process might be related to cancer aggressiveness and metastasis (29-31). Moreover, involvement of EMT in drug resistance has been reported, and inhibition of EMT reverses drug resistance (32-34). Therefore, in considering E-cadherin expression status, it would be interesting to investigate whether, after initial endocrine-based therapy, the tumor profiles after eribulin treatment are more favorable to cancer patients than those after capecitabine. In addition, the molecular mechanisms of regulation of Ecadherin by eribulin are unknown, and further studies are required to reveal them.

In conclusion, we demonstrated the antitumor activity of eribulin and capecitabine after fulvestrant plus palbociclib therapy in luminal-type BC PDX models, and we showed that eribulin had significantly greater activity than capecitabine in the luminal-type BC PDX OD-BRE-0438 model. Furthermore, eribulin increased E-cadherin expression on the tumor cell membrane, whereas capecitabine decreased it relative to eribulin, demonstrating that eribulin given after endocrine therapy might promote a favorable tumor microenvironment. Our findings provide preclinical evidence for the eribulin treatment of luminal-type $\mathrm{BC}$ in patients who are resistant to endocrine therapy plus CDK4/6 inhibitors.

\section{Conflicts of Interest}

Y.N., M.A., T.N., T.S., and Y.F. were employees of Eisai Co., Ltd. at the time the study was performed. D.F. is an employee of Oncodesign.

\section{Authors' Contributions}

Y.N., M.A., T.N., T.S., and Y.F. designed the study. Y.N., M.A., T.N., and D.F. collected the data. Y.N. drafted the manuscript. All authors discussed the results and contributed to the final manuscript.

\section{Acknowledgements}

The Authors gratefully acknowledge Caroline Mignard, Francis Bichat, Olivier Duchamp, Marc Hillairet de Boisferon (Oncodesign), and Sunplanet Co., Ltd. for their excellent technical support. The Authors also thank their colleagues at Tsukuba Research Laboratories in Eisai Co., Ltd. for their fruitful discussions and constructive comments.

\section{References}

1 Finn RS, Crown JP, Lang I, Boer K, Bondarenko IM, Kulyk SO, Ettl J, Patel R, Pinter T, Schmidt M, Shparyk Y, Thummala AR, Voytko NL, Fowst C, Huang X, Kim ST, Randolph S and Slamon DJ: The cyclin-dependent kinase 4/6 inhibitor palbociclib in combination with letrozole versus letrozole alone 
as first-line treatment of estrogen receptor-positive, HER2negative, advanced breast cancer (PALOMA-1/TRIO-18): a randomised phase 2 study. Lancet Oncol 16(1): 25-35, 2015. PMID: 25524798. DOI: 10.1016/S1470-2045(14)71159-3

2 Cristofanilli M, Turner NC, Bondarenko I, Ro J, Im SA, Masuda N, Colleoni M, DeMichele A, Loi S, Verma S, Iwata H, Harbeck N, Zhang K, Theall KP, Jiang Y, Bartlett CH, Koehler M and Slamon D: Fulvestrant plus palbociclib versus fulvestrant plus placebo for treatment of hormone-receptor-positive, HER2-negative metastatic breast cancer that progressed on previous endocrine therapy (PALOMA-3): final analysis of the multicentre, double-blind, phase 3 randomised controlled trial. Lancet Oncol 17(4): 425-439, 2016. PMID: 26947331. DOI: 10.1016/S1470-2045(15)00613-0

3 Hortobagyi GN, Stemmer SM, Burris HA, Yap YS, Sonke GS, Paluch-Shimon S, Campone M, Blackwell KL, André F, Winer EP, Janni W, Verma S, Conte P, Arteaga CL, Cameron DA, Petrakova K, Hart LL, Villanueva C, Chan A, Jakobsen E, Nusch A, Burdaeva O, Grischke EM, Alba E, Wist E, Marschner N, Favret AM, Yardley D, Bachelot T, Tseng LM, Blau S, Xuan F, Souami F, Miller M, Germa C, Hirawat S and O'Shaughnessy J: Ribociclib as first-line therapy for HR-positive, advanced breast cancer. N Engl J Med 375(18): 1738-1748, 2016. PMID: 27717303. DOI: 10.1056/NEJMoa1609709

4 Sledge GW Jr, Toi M, Neven P, Sohn J, Inoue K, Pivot X, Burdaeva O, Okera M, Masuda N, Kaufman PA, Koh H, Grischke EM, Frenzel M, Lin Y, Barriga S, Smith IC, Bourayou $\mathrm{N}$ and Llombart-Cussac A: MONARCH 2: Abemaciclib in combination with fulvestrant in women with HR+/HER2advanced breast cancer who had progressed while receiving endocrine therapy. J Clin Oncol 35(25): 2875-2884, 2017. PMID: 28580882. DOI: 10.1200/JCO.2017.73.7585

5 Goetz MP, Toi M, Campone M, Sohn J, Paluch-Shimon S, Huober J, Park IH, Trédan O, Chen SC, Manso L, Freedman OC, Garnica Jaliffe G, Forrester T, Frenzel M, Barriga S, Smith IC, Bourayou $\mathrm{N}$ and Di Leo A: MONARCH 3: Abemaciclib as initial therapy for advanced breast cancer. J Clin Oncol 35(32): 3638-3646, 2017. PMID: 28968163. DOI: 10.1200/JCO.2017.75.6155

6 Wang Y, Habgood GJ, Christ WJ, Kishi Y, Littlefield BA and Yu MJ: Structure-activity relationships of halichondrin B analogues: modifications at C.30-C.38. Bioorg Med Chem Lett 10(10): 1029-1032, 2000. PMID: 10843209. DOI: 10.1016/s0960$894 x(00) 00150-5$

7 Towle MJ, Salvato KA, Budrow J, Wels BF, Kuznetsov G, Aalfs KK, Welsh S, Zheng W, Seletsky BM, Palme MH, Habgood GJ, Singer LA, Dipietro LV, Wang Y, Chen JJ, Quincy DA, Davis A, Yoshimatsu K, Kishi Y, Yu MJ and Littlefield BA: In vitro and in vivo anticancer activities of synthetic macrocyclic ketone analogues of halichondrin B. Cancer Res 61(3): 1013-1021, 2001. PMID: 11221827.

8 Jordan MA, Kamath K, Manna T, Okouneva T, Miller HP, Davis C, Littlefield BA and Wilson L: The primary antimitotic mechanism of action of the synthetic halichondrin E7389 is suppression of microtubule growth. Mol Cancer Ther 4(7): 1086-1095, 2005. PMID: 16020666. DOI: 10.1158/1535-7163.MCT-04-0345

9 Cortes J, O'Shaughnessy J, Loesch D, Blum JL, Vahdat LT, Petrakova K, Chollet P, Manikas A, Diéras V, Delozier T, Vladimirov V, Cardoso F, Koh H, Bougnoux P, Dutcus CE, Seegobin S, Mir D, Meneses N, Wanders $J$ and Twelves C: Eribulin monotherapy versus treatment of physician's choice in patients with metastatic breast cancer (EMBRACE): a phase 3 open-label randomised study. Lancet 377(9769): 914-923, 2011. PMID: 21376385. DOI: 10.1016/S0140-6736(11)60070-6

10 Funahashi Y, Okamoto K, Adachi Y, Semba T, Uesugi M, Ozawa Y, Tohyama O, Uehara T, Kimura T, Watanabe H, Asano M, Kawano S, Tizon X, McCracken PJ, Matsui J, Aoshima K, Nomoto $\mathrm{K}$ and Oda $\mathrm{Y}$ : Eribulin mesylate reduces tumor microenvironment abnormality by vascular remodeling in preclinical human breast cancer models. Cancer Sci 105(10): 1334-1342, 2014. PMID: 25060424. DOI: 10.1111/cas.12488

11 Yoshida T, Ozawa Y, Kimura T, Sato Y, Kuznetsov G, Xu S, Uesugi M, Agoulnik S, Taylor N, Funahashi Y and Matsui J: Eribulin mesilate suppresses experimental metastasis of breast cancer cells by reversing phenotype from epithelialmesenchymal transition (EMT) to mesenchymal-epithelial transition (MET) states. Br J Cancer 110(6): 1497-1505, 2014. PMID: 24569463. DOI: 10.1038/bjc.2014.80

12 DeRose YS, Wang G, Lin YC, Bernard PS, Buys SS, Ebbert MT, Factor R, Matsen C, Milash BA, Nelson E, Neumayer L, Randall RL, Stijleman IJ, Welm BE and Welm AL: Tumor grafts derived from women with breast cancer authentically reflect tumor pathology, growth, metastasis and disease outcomes. Nat Med 17(11): 1514-1520, 2011. PMID: 22019887. DOI: 10.1038/nm.2454

13 Asano M, Matsui J, Towle MJ, Wu J, McGonigle S, DE Boisferon MH, Uenaka T, Nomoto K and Littlefield BA: Broadspectrum preclinical antitumor activity of eribulin (Halaven ${ }^{\circledR}$ ): combination with anticancer agents of differing mechanisms. Anticancer Res 38(6): 3375-3385, 2018. PMID: 29848686. DOI: 10.21873/anticanres.12604

14 Innovative Models Initiative Against Cancer. Available at: http://www.imodi-cancer.com

15 Gao H, Korn JM, Ferretti S, Monahan JE, Wang Y, Singh M, Zhang C, Schnell C, Yang G, Zhang Y, Balbin OA, Barbe S, Cai H, Casey F, Chatterjee S, Chiang DY, Chuai S, Cogan SM, Collins SD, Dammassa E, Ebel N, Embry M, Green J, Kauffmann A, Kowal C, Leary RJ, Lehar J, Liang Y, Loo A, Lorenzana E, Robert McDonald E $3^{\text {rd }}$, McLaughlin ME, Merkin J, Meyer R, Naylor TL, Patawaran M, Reddy A, Röelli C, Ruddy DA, Salangsang F, Santacroce F, Singh AP, Tang Y, Tinetto W, Tobler S, Velazquez R, Venkatesan K, Von Arx F, Wang HQ, Wang Z, Wiesmann M, Wyss D, Xu F, Bitter H, Atadja P, Lees E, Hofmann F, Li E, Keen N, Cozens R, Jensen MR, Pryer NK, Williams JA and Sellers WR: High-throughput screening using patient-derived tumor xenografts to predict clinical trial drug response. Nat Med 21(11): 1318-1125, 2015. PMID: 26479923. DOI: $10.1038 / \mathrm{nm} .3954$

16 Babraham Bioinformatics: FastQC. Available at: http://www. bioinformatics.babraham.ac.uk/projects/fastqc/

17 Bolger AM, Lohse M and Usadel B: Trimmomatic: a flexible trimmer for Illumina sequence data. Bioinformatics 30(15): 21142120, 2014. PMID: 24695404. DOI: 10.1093/bioinformatics/ btu 170

18 Dobin A, Davis CA, Schlesinger F, Drenkow J, Zaleski C, Jha S, Batut P, Chaisson $M$ and Gingeras TM: STAR: ultrafast universal RNA-seq aligner. Bioinformatics 29(1): 15-21, 2013. PMID: 23104886. DOI: 10.1093/bioinformatics/bts635

19 Trapnell C, Roberts A, Goff L, Pertea G, Kim D, Kelley DR, Pimentel H, Salzberg SL, Rinn JL and Pachter L: Differential gene and transcript expression analysis of RNA-seq experiments with TopHat and Cufflinks. Nat Protoc 7(3): 562-578, 2012. PMID: 22383036. DOI: 10.1038/nprot.2012.016 
20 Mougalian SS, Feinberg BA, Wang E, Alexis K, Chatterjee D, Knoth RL, Nero D, Miller T, Liassou D and Kish JK: Observational study of clinical outcomes of eribulin mesylate in metastatic breast cancer after cyclin-dependent kinase 4/6 inhibitor therapy. Future Oncol 15(34): 3935-3944, 2019. PMID: 31660764. DOI: $10.2217 /$ fon-2019-0537

21 Qin G, Xu F, Qin T, Zheng Q, Shi D, Xia W, Tian Y, Tang Y, Wang J, Xiao X, Deng W and Wang S: Palbociclib inhibits epithelial-mesenchymal transition and metastasis in breast cancer via c-Jun/COX-2 signaling pathway. Oncotarget 6(39): 4179441808, 2015. PMID: 26540629. DOI: 10.18632/oncotarget.5993

22 Liu T, Yu J, Deng M, Yin Y, Zhang H, Luo K, Qin B, Li Y, Wu C, Ren T, Han Y, Yin P, Kim J, Lee S, Lin J, Zhang L, Zhang J, Nowsheen S, Wang L, Boughey J, Goetz MP, Yuan J and Lou Z: CDK4/6-dependent activation of DUB3 regulates cancer metastasis through SNAIL1. Nat Commun 8: 13923, 2017. PMID: 28067227. DOI: 10.1038/ncomms 13923

23 Rencuzogulları O, Yerlikaya PO, Gürkan AÇ, Arısan ED and Telci D: Palbociclib, a selective CDK4/6 inhibitor, restricts cell survival and epithelial-mesenchymal transition in Panc-1 and MiaPaCa-2 pancreatic cancer cells. J Cell Biochem 121(1): 508523, 2020. PMID: 31264276. DOI: 10.1002/jcb.29249

24 Zhang Z, Li J, Ou Y, Yang G, Deng K, Wang Q, Wang Z, Wang W, Zhang Q, Wang H, Sun W, Sun P and Yang S: CDK4/6 inhibition blocks cancer metastasis through a USP51-ZEB1dependent deubiquitination mechanism. Signal Transduct Target Ther 5(1): 25, 2020. PMID: 32296027. DOI: $10.1038 / \mathrm{s} 41392-$ 020-0118-x

25 Zhang W, Feng M, Zheng G, Chen Y, Wang X, Pen B, Yin J, Yu $\mathrm{Y}$ and $\mathrm{He} \mathrm{Z}$ : Chemoresistance to 5-fluorouracil induces epithelialmesenchymal transition via up-regulation of Snail in MCF7 human breast cancer cells. Biochem Biophys Res Commun 417(2): 679685, 2012. PMID: 22166209. DOI: 10.1016/j.bbrc.2011.11.142

26 Terashima M, Sakai K, Togashi Y, Hayashi H, De Velasco MA, Tsurutani J and Nishio K: Synergistic antitumor effects of S-1 with eribulin in vitro and in vivo for triple-negative breast cancer cell lines. Springerplus 3: 417, 2014. PMID: 25140293. DOI: 10.1186/2193-1801-3-417

27 Kitahara H, Hirai M, Kato K, Bou-Gharios G, Nakamura H and Kawashiri S: Eribulin sensitizes oral squamous cell carcinoma cells to cetuximab via induction of mesenchymal-to-epithelial transition. Oncol Rep 36(6): 3139-3144, 2016. PMID: 27779690. DOI: $10.3892 /$ or.2016.5189
28 Kashiwagi S, Asano Y, Goto W, Takada K, Takahashi K, Hatano T, Tanaka S, Takashima T, Tomita S, Motomura H, Ohsawa M, Hirakawa K and Ohira M: Mesenchymal-epithelial transition and tumor vascular remodeling in eribulin chemotherapy for breast cancer. Anticancer Res 38(1): 401-410, 2018. PMID: 29277801. DOI: 10.21873 /anticanres.12236

29 Frixen UH, Behrens J, Sachs M, Eberle G, Voss B, Warda A, Löchner D and Birchmeier W: E-cadherin-mediated cell-cell adhesion prevents invasiveness of human carcinoma cells. J Cell Biol 113(1): 173-185, 1991. PMID: 2007622. DOI: 10.1083/ jcb.113.1.173

30 Derksen PW, Liu X, Saridin F, van der Gulden H, Zevenhoven J, Evers B, van Beijnum JR, Griffioen AW, Vink J, Krimpenfort P, Peterse JL, Cardiff RD, Berns A and Jonkers J: Somatic inactivation of E-cadherin and p53 in mice leads to metastatic lobular mammary carcinoma through induction of anoikis resistance and angiogenesis. Cancer Cell 10(5): 437-449, 2006. PMID: 17097565. DOI: 10.1016/j.ccr.2006.09.013

31 Onder TT, Gupta PB, Mani SA, Yang J, Lander ES and Weinberg RA: Loss of E-cadherin promotes metastasis via multiple downstream transcriptional pathways. Cancer Res 68(10): 36453654, 2008. PMID: 18483246. DOI: 10.1158/0008-5472.CAN-072938

32 Du B and Shim JS: Targeting epithelial-mesenchymal transition (EMT) to overcome drug resistance in cancer. Molecules 21(7): 965, 2016. PMID: 27455225. DOI: 10.3390/molecules21070965

33 Uramoto H, Iwata T, Onitsuka T, Shimokawa H, Hanagiri T and Oyama T: Epithelial-mesenchymal transition in EGFR-TKI acquired resistant lung adenocarcinoma. Anticancer Res 30(7): 2513-2517, 2010. PMID: 20682976.

34 Toden S, Okugawa Y, Jascur T, Wodarz D, Komarova NL, Buhrmann C, Shakibaei M, Boland CR and Goel A: Curcumin mediates chemosensitization to 5-fluorouracil through miRNAinduced suppression of epithelial-to-mesenchymal transition in chemoresistant colorectal cancer. Carcinogenesis 36(3): 355-367, 2015. PMID: 25653233. DOI: 10.1093/carcin/bgv006

Received October 30, 2020

Revised November 15, 2020 Accepted November 16, 2020 